Population Study

Oral

Abstract ID: 133

\title{
Absorbance value in middle-aged and older adult population using wideband tympanometry
}

\author{
Nurul Atiqah Mohd Zaki | Marina L. Alisa Puteri \\ Kulliyyah of Allied Health Sciences, International Islamic University Malaysia
}

Introduction: Wideband tympanometry is a new version of tympanometry available for audiology clinics. The scarcity of information on middle-aged and older adult population leads to study. This study focuses on ear effect, tests re-test reliability, measurement of absorbance according to different age group and comparing current study finding with established normative. Methods: 27 participants (15 men, 12 women, and aged 50-73 years) which comprised of 13 adults with age more than 50 years, 10 adults that aged more than 60 years and 4 adults that aged more than 70 years were assessed using wideband tympanometry. Energy absorbance data were collected at 15 frequencies using a tympanometry developed by Interacoustics. Each side of the ear of the participants was measured three times. Results: An independent t-test and repeated measure ANOVA was done respectively showed that there is no variability observes on ear effect and test re-test reliability. Measurement of absorbance according to age group showed significant difference in middle-aged adults ( 50 years old group and 60 years old age group, $Z=-2.593, p=0.03$ ) while no significant differences observed between middle-aged and older adults. The mean of absorbance was found to be higher than the other studies at $257 \mathrm{~Hz}$ until $1587 \mathrm{~Hz}$ and pattern were reverse for frequency $3174 \mathrm{~Hz}$ above. Conclusions: This study provides evidence of ear effect, test re-test reliability, and age of measurement of absorbance in middle-aged and older adult population. This finding supports the important establishing age specific norm starting from the middle-aged population.

KEYWORDS: Wideband tympanometry, middle ear, ear effect, test-retest reliability, age 